This item was submitted to Loughborough's Research Repository by the author.

Items in Figshare are protected by copyright, with all rights reserved, unless otherwise indicated.

\title{
Fuzzy logic control for mobile service systems with limited resources
}

PLEASE CITE THE PUBLISHED VERSION

PUBLISHER

Professional Engineering Publishing / @ IMECHE

VERSION

VoR (Version of Record)

LICENCE

CC BY-NC-ND 4.0

REPOSITORY RECORD

Othman, Fadel M.Y., and Robert M. Parkin. 2019. "Fuzzy Logic Control for Mobile Service Systems with Limited Resources”. figshare. https://hdl.handle.net/2134/4720. 
This item was submitted to Loughborough's Institutional Repository (https://dspace.lboro.ac.uk/) by the author and is made available under the following Creative Commons Licence conditions.

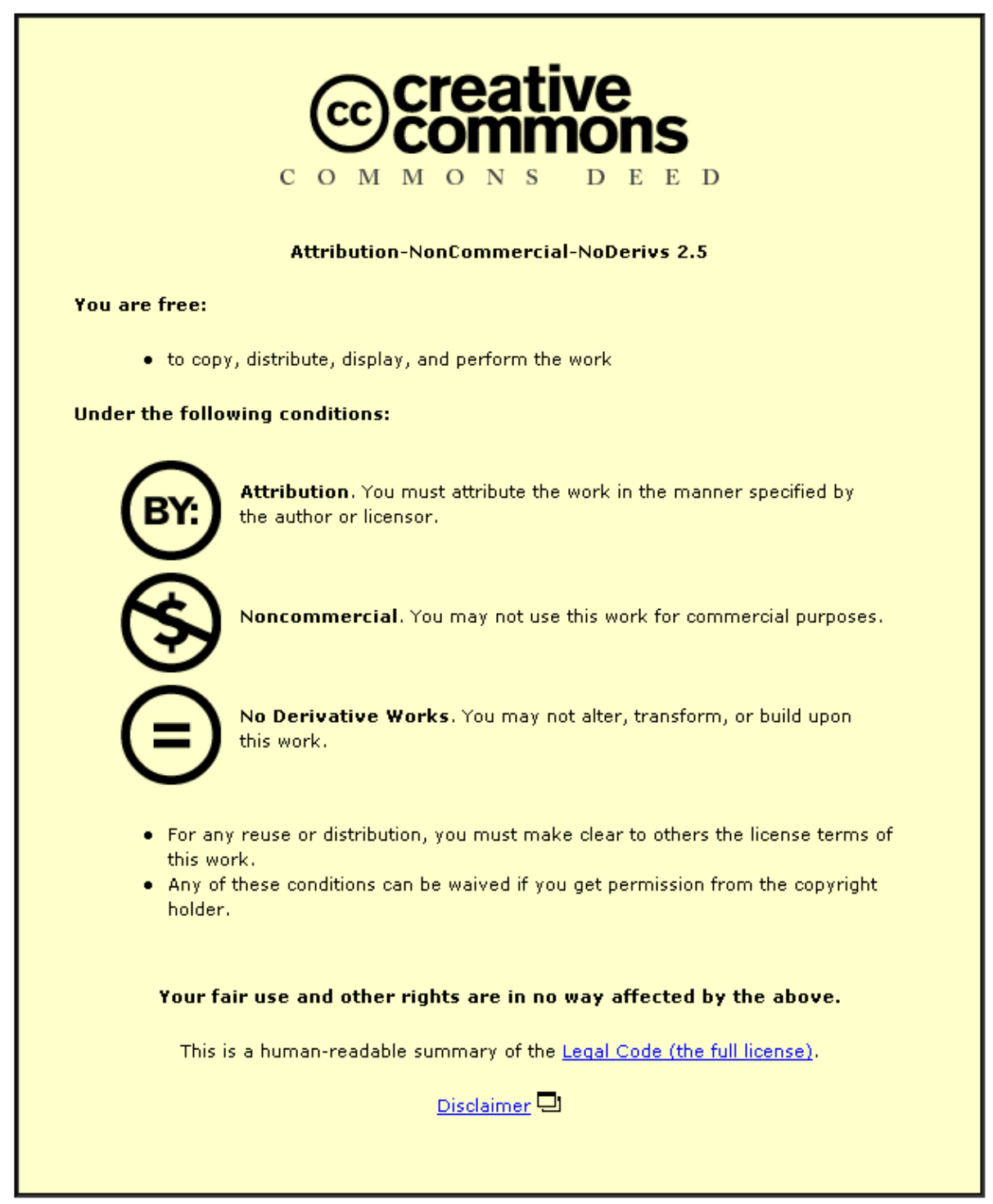

For the full text of this licence, please go to: http://creativecommons.org/licenses/by-nc-nd/2.5/ 


\title{
Fuzzy logic control for mobile service systems with limited resources
}

\author{
F Othman ${ }^{1,2 *}$ and $\mathbf{R}$ M Parkin ${ }^{1}$ \\ ${ }^{1}$ Holywell Mechatronics Research Centre, Wolfson School of Mechanical and Manufacturing Engineering, \\ Loughborough University, Loughborough, Leicestershire, UK \\ ${ }^{2}$ The Custodian of the Two Holy Mosques Institute for Hajj Research, Umm Al-Qura University, Makkah, Saudi \\ Arabia
}

\begin{abstract}
It is common to control systems or plants through changing the values of their inputs in continuous or small discrete steps to achieve gradual change of the performance of the plant under control. However, when changing the inputs can only be achieved in large discrete values, problems arise on achieving stability of the plant under control as such large discrete changes in inputs cause large overshoots and oscillation at the outputs. More difficulties arise when multiple plants are competing simultaneously for limited resources of such large discrete values.

This paper describes an approach for controlling such plants. The case study presented is to control a proposed system of mobile service units (MSUs) to be used in a mega-event, the Hajj, where different locations/zones will be competing simultaneously for a limited number of MSUs in order to provide adequate services for their continuously changing number of visitors. These MSUs are of high discrete values such that their provision or removal generates a significant change in the performance of the system.

Fuzzy logic controllers (FLCs), representing each zone, are developed for issuing requests for MSUs based on their local situations. These requests are evaluated by the control centre using other sets of FLCs to consider global situations. An algorithm is used to respond to these evaluated requests according to their relative importance and issues orders to MSUs to move to the right zone at the right time to provide the appropriate amount of service for the existing number of visitors or what can be called 'just-in-time MSUs' (JIT-MSUs). Simulation results show that such a system can be controlled using the approach suggested by this paper and the response of the system varies with the values of its discrete inputs. Some conclusions and recommendations are drawn for the appropriate discrete input values and their control settings. The procedure for evaluating the different simulation runs for the different control settings is also presented.
\end{abstract}

Keywords: fuzzy logic controllers, discrete inputs of high values, competing plants, limited resources, decision making, artificial intelligence, services for the Hajj

\section{BACKGROUND}

The Hajj, the annual Muslim pilgrimage to Makkah, Saudi Arabia, is a mega-event that attracts millions of visitors/pilgrims from all over the world for about two weeks. In order to host the 4.8 millions visitors expected by year 2025 , a new master plan [1] is proposed for the

The MS was received on 6 June 2003 and was accepted after revision for publication on 20 October 2003.

* Corresponding author: Holywell Mechatronics Research Centre, Wolfson School of Mechanical and Manufacturing Engineering, School of Engineering, Loughborough University, Loughborough, Leicestershire LE11 $3 T U, U K$.

I05803 C IMechE 2004 four cities of the Hajj: Makkah, Mina, Muzdalifah and Arafat. The concept of the master plan is based on six main roads or loops, each loop serves four zones at each city and each zone accommodates 200000 pilgrims, giving a total capacity of 4.8 million.

Due to the short stay of pilgrims at the four cities during the Hajj (Fig. 1), which lasts for a few days every year, it would be too expensive to repeat fixed service centres at the four cities, as it makes the total service capacity enough for 19.2 million while serving only 4.8 million at a time. Therefore, in order to reduce the cost and improve the quality of services provided, a system of mobile service units (MSUs), originally suggested 


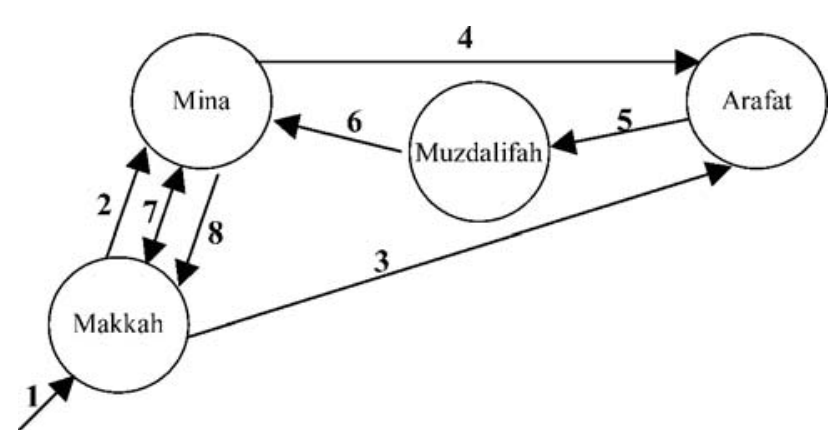

Fig. 1 The movements of pilgrims about the four cities during the Hajj

in references [2] and [3] and previously modelled in reference [4], is proposed for the new master plan to provide mobile services instead of fixed service centres. Being mobile, these MSUs could serve at the right place whenever needed without being limited to specific locations or seasons. However, the management/control of the movements of these MSUs to satisfy the varying demands at the different locations/zones is quite challenging, especially when considering traffic limitations.

\section{THE CONTROL PROBLEM}

The mass movement of visitors between the different zones at the four cities shifts demand peaks for services from one zone to another and need to be satisfactorily fulfilled by MSUs. The system to be controlled is the process of providing services for pilgrims during the Hajj using MSUs. The change in demand for services (or the controlled variable) is continuous while the response of the control, by sending or returning MSUs (or the manipulated variable), can only be achieved in large discrete values, which cause severe overshooting and disturbance to the status of services provided (or the error). The control problem is to make the right response to these variable demands at the different competing zones, putting into context the limited resources and traffic conditions on roads of the MSUs in order to give priority not to hinder the important movements of visitors during the Hajj nor to cause severe shortage of services provided for them.

\section{THE DEVELOPED CONTROL FOR THE SYSTEM OF MSUS}

Fuzzy logic controllers (FLC) are chosen to control the system of MSUs due to their ability to represent the verbal control rules of service providers, to deal with the non-linearity of the system and to demonstrate their high tolerance to imprecision without the need for an explicit mathematical modelling of the system $[\mathbf{5}, \mathbf{6}]$. FLCs showed successful implementation in applications that, to some extent, have some similarity in nature to parts of the system of MSUs, such as controlling an automated goods transporting device in a distribution centre [7], controlling multiple vehicles [8] and solving the problem of period length selection [9]. Decentralized and hierarchical control are used to organize the control of the system. It is used in reference [10] to control a largescale discrete event system and in reference [11] to show how a coordinator controls the lower-level supervisors in the form of supervisor conjunction.

The developed control for the system of MSUs is derived from the general model of the system [12] and consists of three stages, as shown in Fig. 2. In stage 1, each zone evaluates its needs for MSUs based on its local situation and issues an MSU request to the control centre using a specially developed FLC 'Request'. These requests represent the microscopic point of view of each zone for their demand for a particular service. In stage 2, all MSU requests from all zones are evaluated simultaneously by the control centre using another specially developed FLC 'Evaluation' assigned for each zone, used to consider the current situations at the support zone and the global situations at the system as a whole.

In stage 3, a specifically written function 'MsuOrders', based in the control centre, sorts the evaluated MSU requests according to their relative importance and then executes the appropriate control actions, based on some control parameters, by releasing MSUs from zones that do not need them, sending MSUs to zones that are desperately in need of them or taking no action. By this response from the control centre, the closed control loop is completed.

\subsection{The first stage: issuing an MSU request}

A subsystem located inside each zone contains the fuzzy logic controller 'Request', which issues a score for returning an existing MSU ( -10 to 0$)$ or a score for requesting an additional one ( 0 to 10 ) based on service conditions inside that zone fed as inputs (fuzzy variables) to the controller. The fuzzy variables, their fuzzy membership functions (MFs) and the output of the FLC 'Request' are represented in tabulated form in Table 1.

Service status is defined in this paper as the difference between supply and demand for service. The first fuzzy variable 'ServiceToCapacity', representing the ratio of the current service status to MSU service capacity, is given three MFs: 'NegativeVeryHigh', 'Ok' and 'PositiveVeryHigh' for describing service situations of undersupply, acceptable and over-supply respectively. The second variable 'PopulationChange' is given three MFs: 


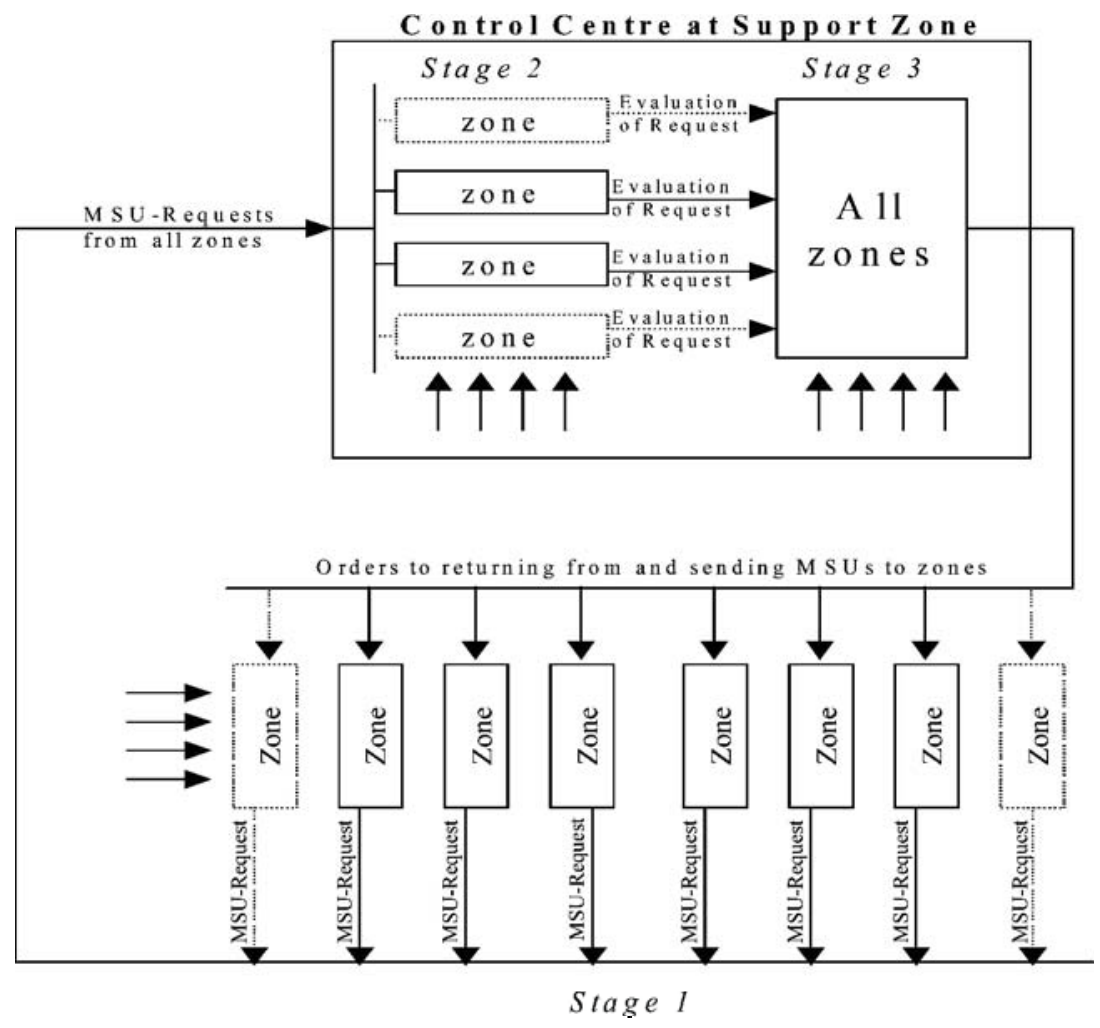

Fig. 2 The three stages for controlling the system of MSUs

Table 1 Fuzzy inputs and output for the FLC 'Request'

\begin{tabular}{lllll}
\hline & & \multicolumn{3}{c}{ 'FutureNeed' } \\
\cline { 3 - 5 } 'ServiceToCapacity' & 'PopulationChange' & 'NegativeHigh' & 'Ok' & 'PositiveHigh' \\
\hline 'NegativeVeryHigh' & 'Falling' & 'Low' & 'NoRequest' & 'NoRequest' \\
& 'NoChange' & 'Urgent' & 'NoRequest' & 'NoRequest' \\
& 'Rising' & 'TopPriority' & 'Low' & 'Low' \\
& 'Falling' & 'NoRequest' & 'NoRequest' & 'NeedVeryLo' \\
& 'NoChange' & 'High' & 'NoRequest' & 'NoRequest' \\
& 'Rising' & 'Urgent' & 'NoRequest' & 'NoRequest' \\
'PositiveVeryHigh' & 'Falling' & 'NoRequest' & 'NeedLo' & 'NotNeededAtAll' \\
& 'NoChange' & 'NoRequest' & 'NoRequest' & 'NeedVeryLo' \\
& 'Rising' & 'Low' & 'NoRequest' & 'NoRequest' \\
\hline
\end{tabular}

'Falling' for describing the situation where there is a decrease in the population when pilgrims are leaving the zone, 'NoChange' when not many pilgrims are coming to the zone or are leaving it and 'Rising' when pilgrims are coming. The third variable 'FutureNeed' is also used to describe the status of the service as the first variable, but for the near future after a predefined period of time 'Lookahead'. The output of the FLC 'MSU-request' is the result of evaluation presented in nine MFs, four of them being 'NotNeededAtAll', 'NotNeeded', 'NeedVeryLo' and 'NeedLo', to describe the degree of need for an existing MSU. One MF, 'NoRequest', reports that the service situation is acceptable and there is no need to return or to send an MSU. The remaining four MFs describe the degree of need for an additional MSU, namely 'Low', 'High', 'Urgent' and 'TopPriority'. The total number of roles due to these three inputs and their MFs is 27, as presented in Table 1.

Figure 3 represents the three inputs and the shapes of their MFs, fuzzy roles in indexed form and the output 'MSU-request' with its nine MFs. The Gaussian shapes for these MFs are used to give a smoother control surface and closer values for inputs about extreme ends of their MFs. The MFs are made overlapped in order to inherit some characteristics from rules applicable to nearby values and to avoid discontinuities in decision making, regions of inputs that fire no rules or sudden requests for MSUs.

Proc. Instn Mech. Engrs Vol. 218 Part I: J. Systems and Control Engineering 


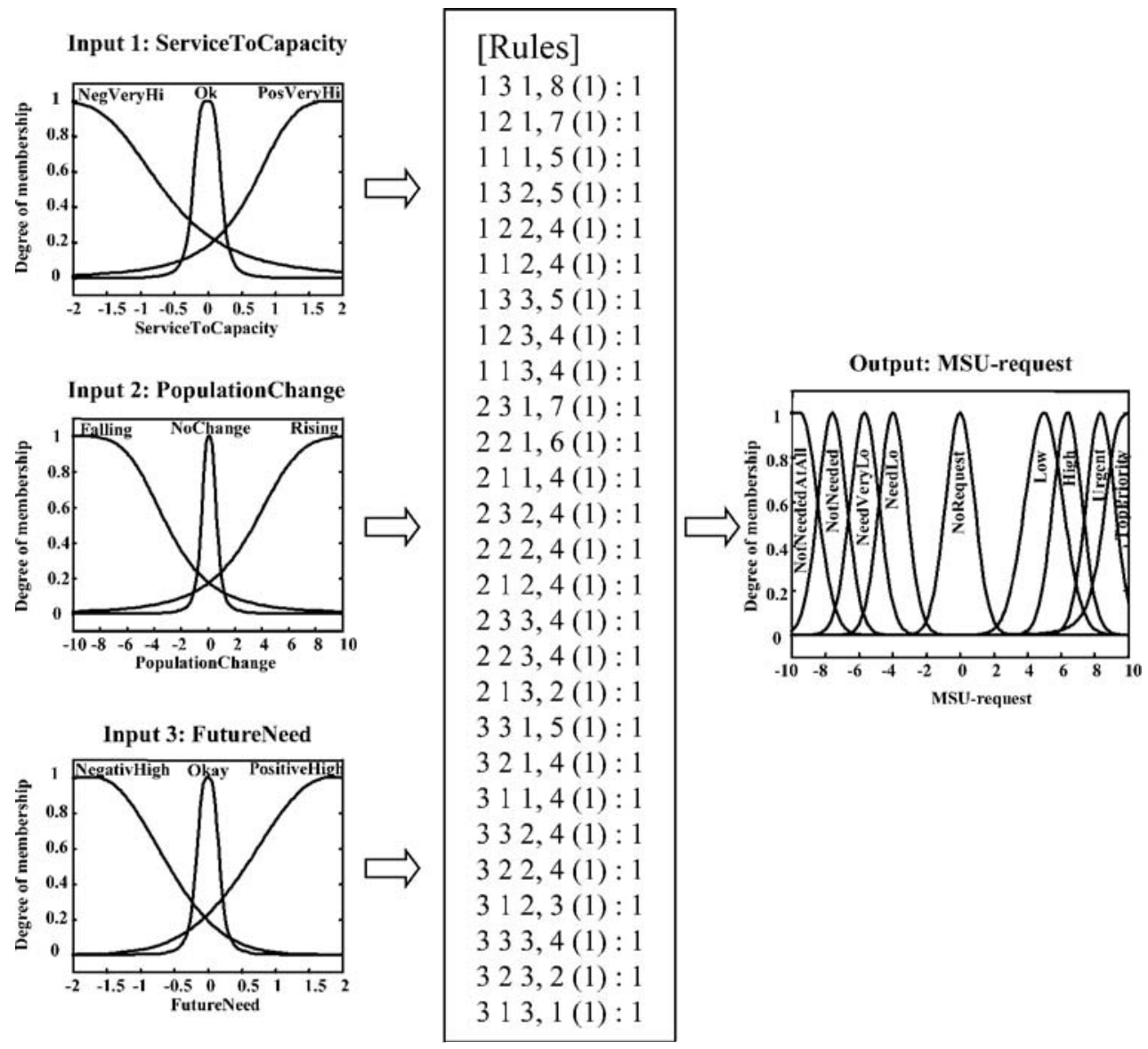

Fig. 3 Fuzzy inputs, rules and output for FLC 'Request'

Figure 4 represents one case study for the evaluation process for the FLC 'Request' given for demonstration. The first input 'ServiceToCapacity' has a value of -0.5 , which indicates that there is a relatively high shortage of service provided, which is equal to half the capacity of an MSU. This value falls only in the region of 'NegativeVeryHigh' for this input; therefore it fires the first nine rules only. The second input 'PopulationChange' indicates that pilgrims are leaving that zone at a relatively slow rate of -0.6 , which has intersections with the three MFs for this input at different values; therefore, some decisions will be inherited from nearby rules. The third input 'FutureNeed' has a value of -0.3 , which indicates that, after a certain time defined by 'Lookahead', if no action is taken, it is expected that there will be still some shortage for service but less than the current situation. The nine rules contribute in some degree or another in the decision making for this case, which can be read as follows: 'Currently there is a shortage in the service provided but pilgrims are leaving the zone and the shortage of the service is expected to be better in the near future; therefore, the resultant evaluation/ request is: there is no severe need for an additional MSU now, but if it could be provided that will improve service in the current stage.' This evaluation is presented in the score of 2.76 (out of 10) for 'MSU-request', which will be reported to the control centre.

\subsection{The second stage: evaluation of MSU requests from all zones}

Requests received from all zones are evaluated simultaneously at the control centre using the FLC 'Evaluation', which considers another set of factors affecting the whole system fed to the controller as inputs. These inputs, their MFs and the rules for this controller are presented in Table 2 and Fig. 5. The first input is the 'MSU-request', received from the zone that this controller evaluates, which is limited to the range -10 to 10 and given five MFs: 'NeedLow' and 'NotNeeded' for unneeded MSUs, 'NoRequest' for good conditions that have no need for returning or sending MSUs and 'HighRequest' and 'Urgent' for the need for an additional MSU. The second input is 'MSU-availability', limited to the range 0 to 1 and given two MFs, 'Available' and 'Rare', to indicate whether too many MSUs are available at the support zone; therefore, most requests can be answered or very few can be answered so only top priority requests can 


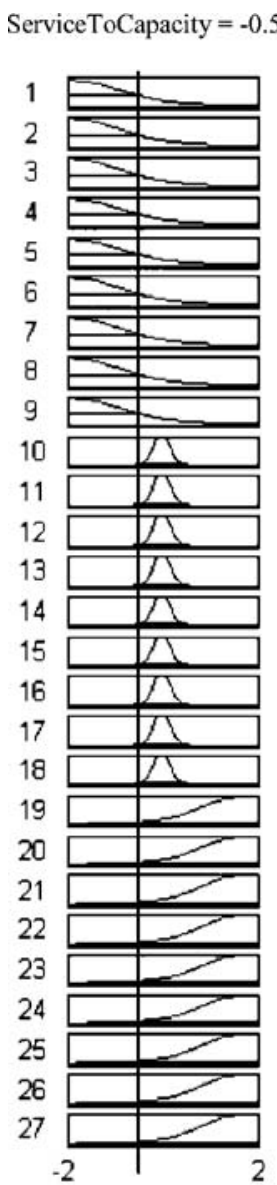

'PopulationChange' $=-0.6$

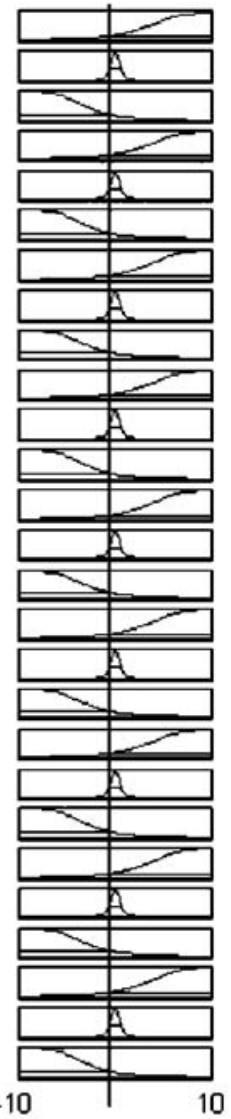

'FutureNeed' $=-0.3$

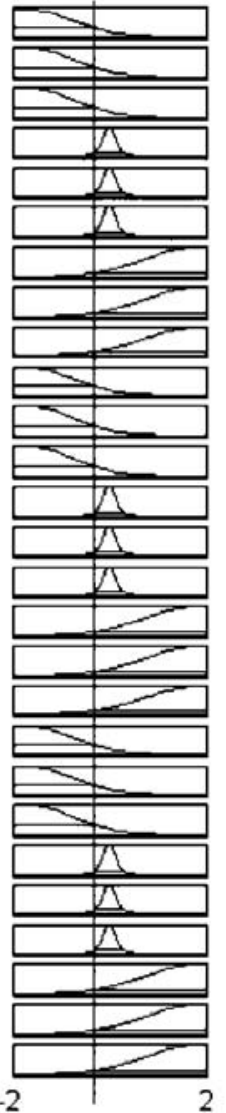

'MSU-request' $=2.76$

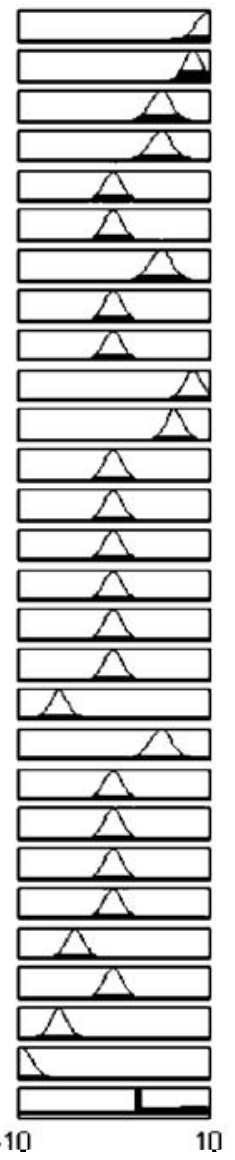

Fig. 4 Implementation of FLC 'Request' for one case study

Table 2 Fuzzy inputs and output for the FLC 'Evaluation'

\begin{tabular}{|c|c|c|c|c|c|}
\hline \multirow[b]{4}{*}{ 'MSU-request' } & \multirow[b]{4}{*}{ 'MSUAvailability' } & \multicolumn{4}{|c|}{ 'CurrentTraffic Volume' } \\
\hline & & \multicolumn{2}{|c|}{ 'TrafficLow' } & \multicolumn{2}{|c|}{ 'TrafficHigh' } \\
\hline & & \multicolumn{4}{|c|}{ 'FutureBusTraffic' } \\
\hline & & 'FewBuses' & ‘ManyBuses’ & 'FewBuses' & 'ManyBuses' \\
\hline \multirow[t]{2}{*}{ 'NotNeeded' } & 'Rare' & 'ReturnMSU' & 'ReturnMSU' & 'NoAction' & 'MayReturned' \\
\hline & 'Available' & 'NoAction' & 'NoAction' & 'NoAction' & 'MayReturned' \\
\hline \multirow[t]{2}{*}{ 'NoRequest' } & 'Rare' & 'NoAction' & 'NoAction' & 'NoAction' & 'NoAction' \\
\hline & 'Available' & 'NoAction' & 'NoAction' & 'NoAction' & 'NoAction' \\
\hline \multirow[t]{2}{*}{ 'Urgent' } & 'Rare' & 'SendUrgent' & 'SendUrgent' & 'NoAction' & 'SendNormal' \\
\hline & 'Available' & 'SendUrgent' & 'SendUrgent' & 'SendNormal' & 'SendUrgent' \\
\hline \multirow[t]{2}{*}{ 'NeedLow' } & 'Rare' & 'MayReturned' & 'MayReturned' & 'NoAction' & 'NoAction' \\
\hline & 'Available' & 'NoÁction' & 'MayReturned' & 'NoAction' & 'NoAction' \\
\hline \multirow[t]{2}{*}{ 'HighRequest' } & 'Rare' & 'NoAction' & 'SendNormal' & 'NoAction' & 'NoAction' \\
\hline & 'Available' & 'SendNormal' & 'SendNormal' & 'NoAction' & 'SendNormal' \\
\hline
\end{tabular}

be dealt with. The third input 'CurrentTrafficVolume', limited to the range 0 to 75 , reflects the current traffic volume in the loop and is given two MFs: 'TrafficLow' to indicate that traffic volume is low so many MSUs can be allowed to move and 'TrafficHigh' to indicate that not much room is left for moving MSUs. The fourth input is 'FutureBusTraffic', limited to the range 0 to 65 , given two MFs to describe the expected traffic volume due to the movement of pilgrims' buses, where 'FewBuses' indicates that in the near future there will be few buses on the road so many MSUs can be moved at that time and 'ManyBuses' to indicate that not many MSUs will 


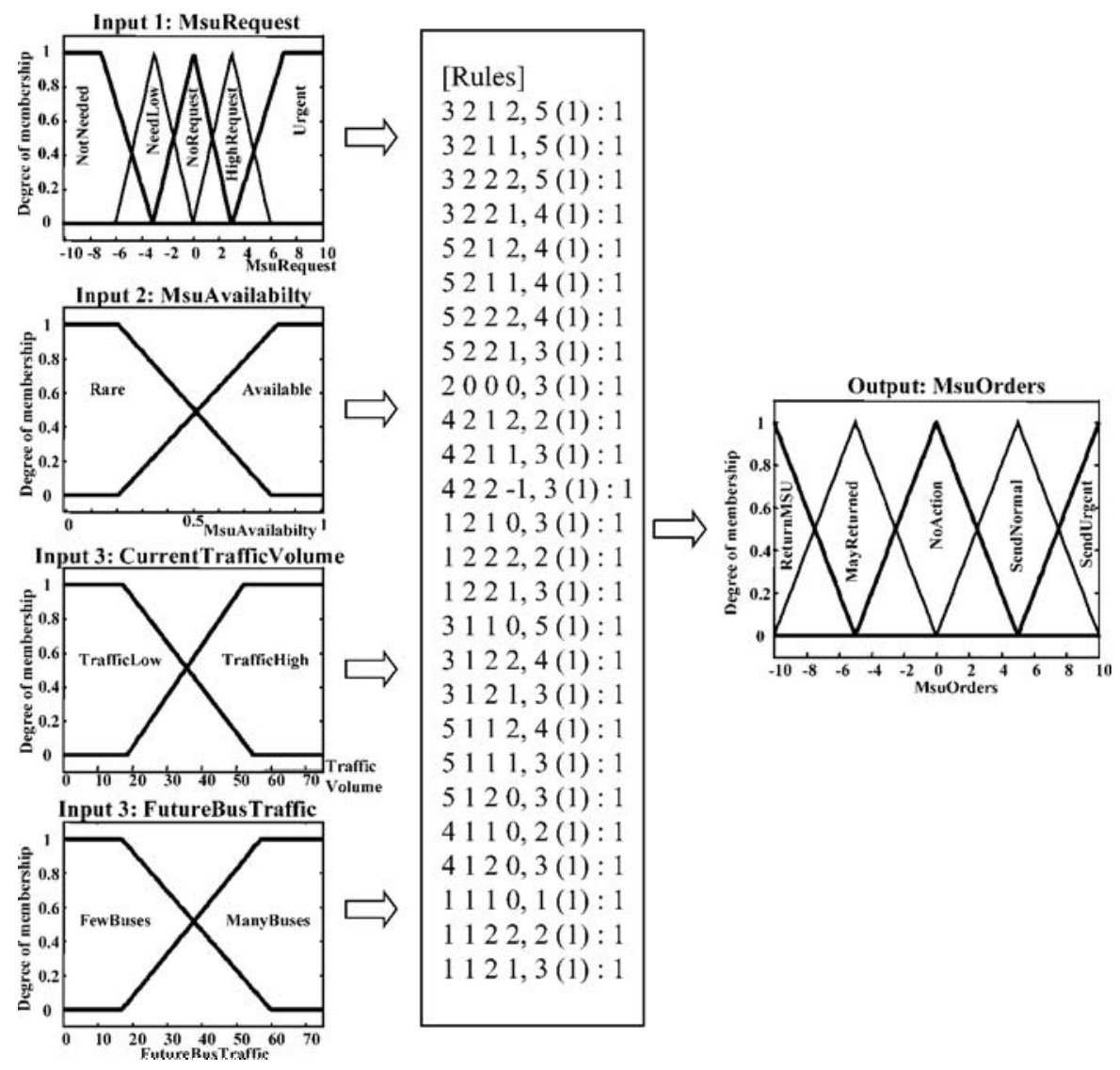

Fig. 5 Fuzzy inputs, rules and output for the fuzzy logic controller 'Evaluation'

be allowed to move after a certain time due to the mass transportation of pilgrims; therefore it should be better to approve that request now.

In order to demonstrate the evaluation process for the FLC 'Evaluation', Fig. 6 shows its implementation for one case study. An 'MSU-request' with a score of 4.9 (out of 10) is issued from a zone, indicating a relatively high need for an additional MSU, which fires the rules that have MFs containing this score. The second input 'MsuAvailabilty' has a value of 0.3 (out of 1), which indicates that relatively not many MSUs are currently available at the support zone. The third input 'CurrentTrafficVolume' with a value of 50 (out of 75) reports that roads are currently crowded, while the fourth input 'FutureBusTraffic' with a value of 22 (out of 65) expects that in the near future not many buses will be on the road. The situation can be read as follows: 'A particular zone has a relatively high need for an additional MSU, but there are few available in the support zone and the roads are too busy now but are expected to be better after some time; therefore, the decision is to wait for better traffic conditions after a while.' This is translated by reducing the score for 'MSU-request' from 4.9 to 1.34. The reduction of the scores of relatively high MSU requests in such situations, of limited resources and busy roads, gives priority to very important MSU requests to be answered.

\subsection{The third stage: issuing orders to sending and returning MSUs}

The specially written MATLAB function 'MsuOrders' sorts the evaluated MSU requests and then issues orders to return unneeded or slightly needed MSUs and sends MSUs to zones according to their relative importance and the global situations for the system as a whole. The inputs for this function are the evaluated 'MSU-request' from all zones, the number of MSUs available at the support zone, the 'Instant-Acceptance-Score', the 'MinAcceptance-Score', the 'Max-Return-Score', the 'InstantReturn-Score', 'Traffic-Volume' and the 'Batch-size' for the MSU service group under investigation. The algorithm for the 'MsuOrders' function can be summarized as follows in conjunction with Fig. 7:

1. Return MSUs from zones scoring below the 'InstantReturn-Score' as they are not needed any more. Stop returning them if the traffic volume approaches the maximum limit, but still make them available for urgent needs.

2. Send MSUs urgently to all zones scoring above the 'Instant-Acceptance-Score' since they need them badly, even by returning MSUs from least needy zones if no MSUs are available at the support zone. Stop sending them if the total traffic volume for buses on the road 

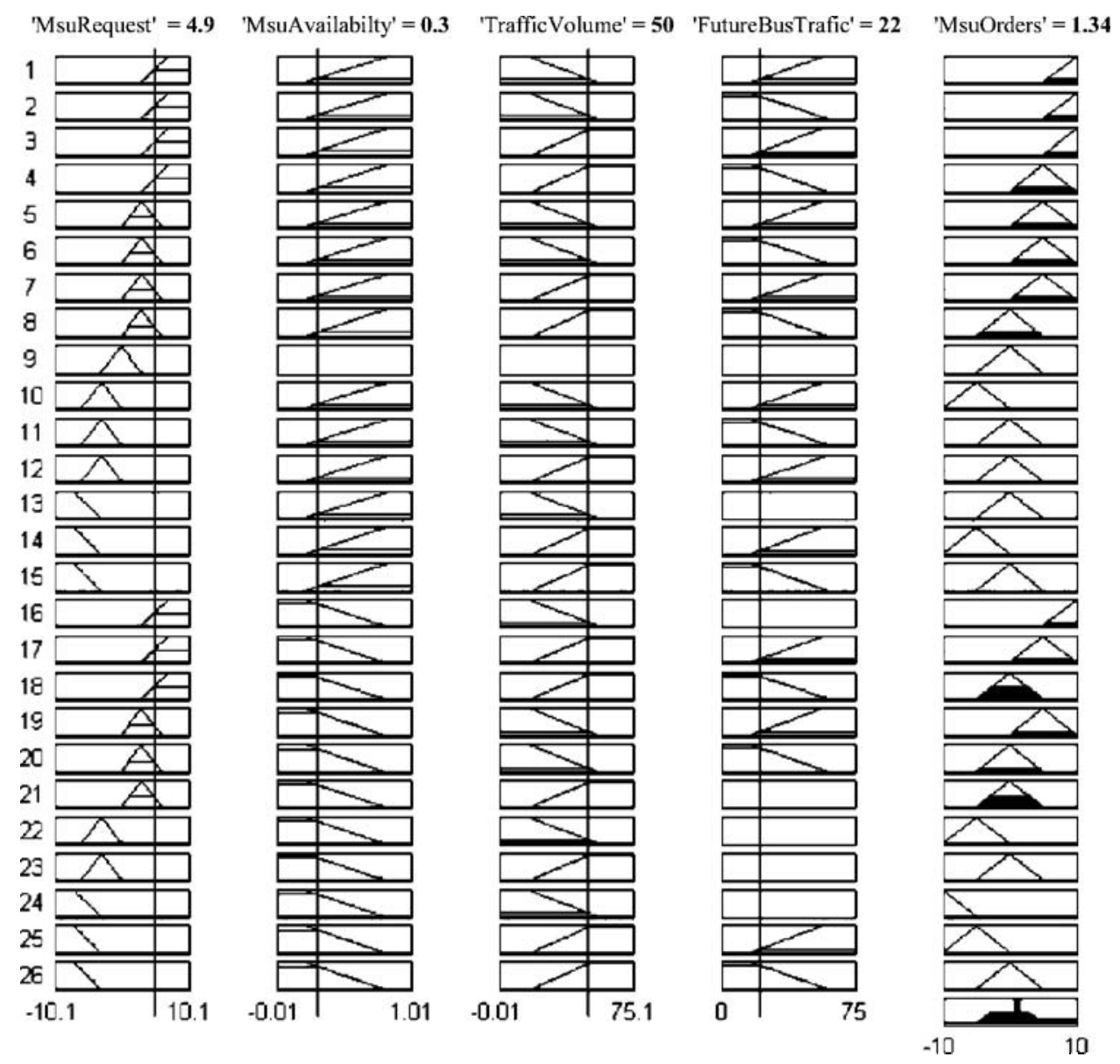

Fig. 6 Implementation of the FLC 'Evaluation' for one case study

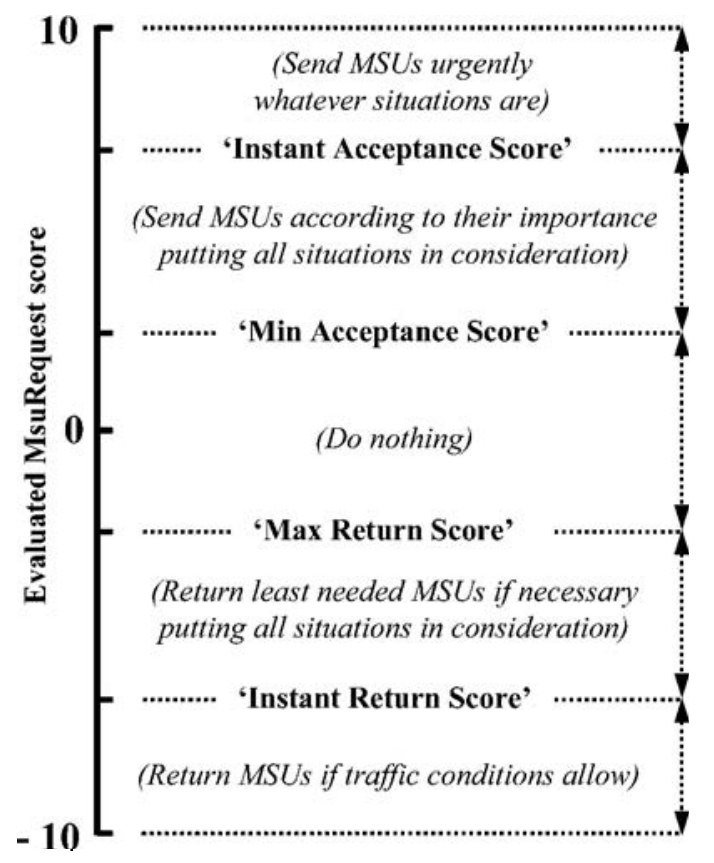

Fig. 7 The different situations for evaluating requests for MSUs and MSUs to be sent exceeds 75 for the large vehicles/ simulation step.

3. Send MSUs to the highest remaining needy zones, in descending order, scoring above the 'Min-AcceptanceScore' and, if necessary, return MSUs from the least needy zones, in ascending order, scoring below the 'Max-Return-Score', provided that it does not contradict the previous 10 orders. In both cases, stop if the total traffic volume exceeds 75 (large vehicles/ simulation step).

4. Take no action for zones scoring above the 'MaxReturn-Score' up to the 'Min-Acceptance-Score' as these zones are in balance between demand and supply of services and sending or releasing MSUs from them will disturb that balance.

\section{SIMULATION RESULTS AND DISCUSSION}

The simulation is set to fixed discrete steps of 1.5 minutes and covers the period from the start of day 7 to the end of day 13 of the month of the Hajj where most activities take place. The different types of MSU are grouped into 
16 groups of similar service capacity and close ranks for speeding up simulation. Each simulation step evaluates one MSU group at a time and the whole 16 groups are re-evaluated every evaluation cycle of 16 simulation steps. The simulation is set to run at different control settings for 'Lookahead' times, 'Max-Return-Score' and 'Min-Acceptance-Score', giving a total of 168 runs. The selection of the ranges for these values are based on previous preliminary simulation runs $[\mathbf{1 2}]$ and are expected to provide acceptable performance for the system.

The discussion starts with defining the stability of the system of MSUs and then presents the procedure for evaluation of simulation runs and the criteria for selection of the appropriate control settings. The main discussion will be based on identifying the appropriate service capacity for MSUs that satisfies the demand for services within the constraints of the system

\subsection{Stability of the system of MSUs}

Different approaches are used to analyse the stability of various fuzzy control systems [13-17]. However, due to the large discrete value for the control action, another approach is used. The instability of the system is considered, in this paper, to occur when an MSU is returned from a zone that it was sent to in any of the 10 previous evaluation cycles and vice versa. Such unstable or inconsistent orders, if they occur, cause confusion to MSU operators and increase traffic volume. The 10 evaluation cycles are equivalent to $4.4 \mathrm{~h}$, which allows any MSU to spend at least $4.4 \mathrm{~h}$ in any location before being assigned to a new job, in order to give commercial MSU operators enough time to settle down and to sell their services. However, this time is not fixed and should be discussed and approved by service supervisors during the planning stage for the Hajj.

In the early versions of the model [12], it was noticed that some unstable MSU orders occur frequently despite the different configurations and functions tried. This is mainly due to the high discrete value for MSU service capacity which, when removed or added to the system, creates high overshoots that disturb the system. In this version of the model, the technique used to achieve stability for MSU requests consists of two steps: firstly, by taking the average of the current and the previous
MSU request to compensate contradicting requests; secondly, by damping the orders to send or to return MSUs by adding conditions to the 'MsuOrders' algorithm. Therefore, if the new order contradicts any of the previous 10 orders, such as returning an MSU that was just sent or vice versa, then no order is issued, as discussed above.

\subsection{Evaluation of simulation results}

The process of evaluation of the 168 simulation runs is based on multiple stages. In the first stage, any control settings that issue contradicting orders within 11 evaluation cycles are rejected. Although the system is made stable for 10 evaluation cycles by the algorithm mentioned before, the rejection is based on the tendency of the system to change status as soon as restrictions are removed. It is found that no single setting can fulfil this condition for all types of MSUs at the same time (Table 3). This is due to the high variation in service capacities of the MSUs, ranging from 13.3 to $200 \mathrm{k}$ inhabitants/pilgrims, showing that appropriate settings for an MSU of low service capacity are not necessarily suitable for an MSU of high service capacity. Therefore, each MSU service group is evaluated separately in order to find out the appropriate control settings for it. When operational, the control centre should use the relevant appropriate control settings for every MSU group under evaluation.

In the second evaluation stage, statistics are calculated for every run (Table 4) in order to present some important issues for determining the appropriate settings for controlling MSUs. The value of the 'Mean-of-ServiceStatus' presents the overall average of service status for a particular service group while the value of the 'Standarddeviation-of-Service-Status' presents how the values of over- and under-supply periods are dispersed; therefore, the closer their values are to zero the better the level of service achieved. The value of the 'Worst-Undersupply-Period' presents the period of the worst incident of shortage of service achieved during simulation and the value of the 'Sum-of-Under-supply-Periods' presents the accumulated shortage of service provided; therefore, one of the objectives of the control should be to minimize their values. The value of the 'Sum-of-Oversupply-Periods' is used to present the capacity of services

Table 3 Number of runs (out of 168 runs) that are considered stable for 11 evaluation cycles

\begin{tabular}{|c|c|c|c|c|c|c|c|c|c|c|c|c|c|c|c|c|}
\hline & \multicolumn{16}{|c|}{ MSU group } \\
\hline & 1 & 2 & 3 & 4 & 5 & 6 & 7 & 8 & 9 & 10 & 11 & 12 & 13 & 14 & 15 & 16 \\
\hline Capacity (pilgrims) (k) & 100 & 100 & 22.2 & 66.7 & 50 & 20 & 25 & 200 & 13.4 & 16.7 & 20 & 20 & 100 & 15.4 & 200 & 50 \\
\hline Stable runs & 168 & 168 & 64 & 168 & 168 & 18 & 49 & 163 & 13 & 8 & 36 & 10 & 168 & 16 & 168 & 168 \\
\hline
\end{tabular}


Table 4 Statistics and evaluation of simulation results for selected MSU groups and their control settings

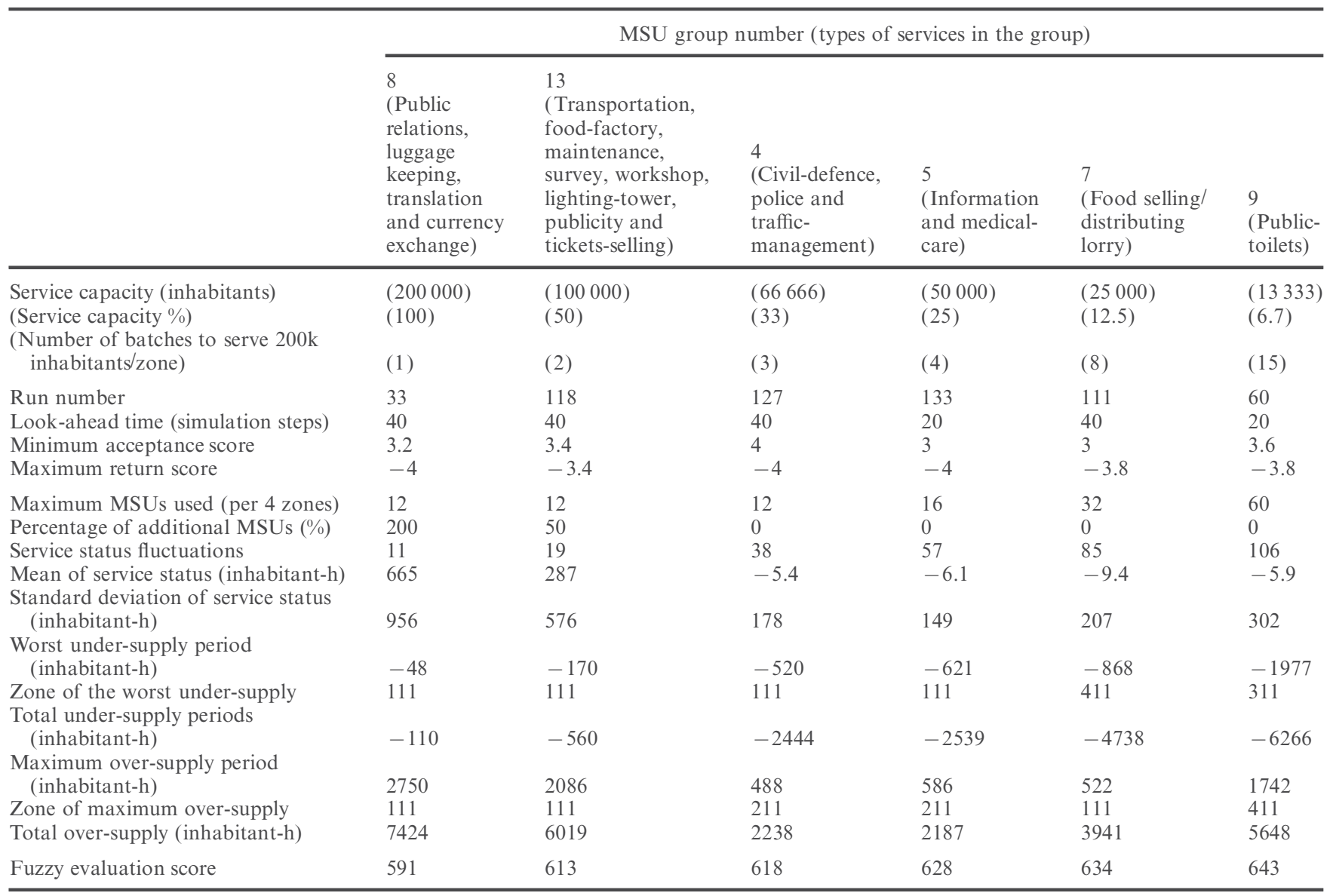

provided but not consumed, which is considered as an economical loss to service providers; therefore, the aims of the control should include minimizing this value for higher efficiency. The value of the 'Maximum-Numberof-MSUs-Used' presents the MSUs resources needed to achieve an acceptable level of service; therefore, reducing its number reduces the capital cost of the system, which is considered as one of the main objectives for using MSUs.

In the third evaluation stage, these statistics are used to compare all runs using a simple FLC for each measure. Each controller has two MFs representing the two extremes of the best and the worst values and the total score is the average of the outputs of these controllers. The total score gives the 'goodness' of each setting relative to others (Table 4). Finally, graphs for runs with the highest scores are plotted, compared and evaluated visually. If an unpreferred behaviour such as short intervals between sending and returning MSUs or long periods of under- or over-supply is detected, the next highest score is plotted and evaluated, and so on, until the appropriate response is found. Table 4 also shows the appropriate control settings selected for some MSU groups and their statistics.

\subsection{MSUs of 'Very-high-service-capacity' and 'High service capacity'}

MSUs of 'Very-high-service-capacity' (VHSC) of 200k inhabitants, such as MSU group 8 shown in Fig. 8, row 1 , require only one unit to serve the whole population of a zone. Therefore, it is either provided to a zone or not. When provided, it fulfils all its needs for that service whatever the population; however, for a low population, the unused service capacity for that unit is reflected as periods of over-supply of the service (Fig. 8, points 1, 2, 4 and 5). On the other hand, if that MSU is removed, the shortage appears as a period of under-supply even for a low population (Fig. 8, point 3 ). It can also be seen in Fig. 8, point 4, that although the first peak of zone 211 reaches less than $100 \mathrm{k}$ inhabitants, or half the capacity of one MSU of group 8, an MSU was needed to cover that need since its absence would have created a severe shortage of service. This explains the need for the 200 per cent additional MSUs (a total of three VHSC MSUs for every four zones) at best. It can be concluded that using VHSC MSUs does not make much reduction in resources for the Hajj compared with fixed service centres, apart from the ability of using them somewhere else outside the Hajj.

Proc. Instn Mech. Engrs Vol. 218 Part I: J. Systems and Control Engineering 

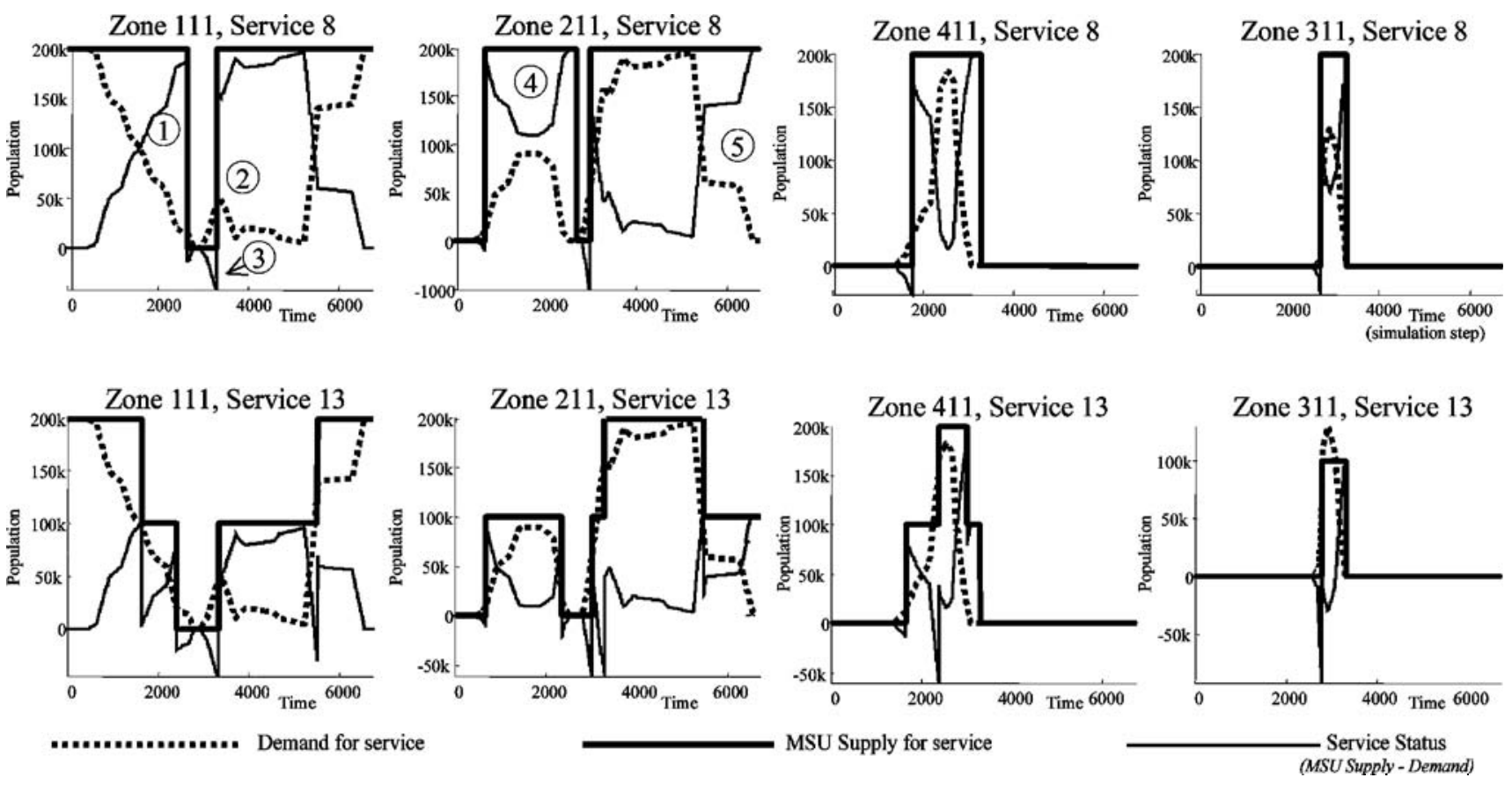

Fig. 8 Response of the control for MSUs of very-high and high service capacities

MSUs of 'High-service-capacity' (HSC) of 100k inhabitants ( 50 per cent of the maximum population of a zone), such as MSU group 13 shown in Fig. 8, row 2, are found to give better results compared with VHSC MSUs such as MSU group 8 discussed above. These improvements can be seen in terms of less over-supply, lower values for mean and standard deviations and, most importantly, in the reduction of the additional MSUs from 200 to 50 per cent respectively.

\subsection{MSUs of 'Medium-service-capacity'}

MSUs of 'Medium-service-capacity' (MSC), such as MSU group 4 shown in Fig. 9, row 1, that have a service capacity of $66.7 \mathrm{k}$ inhabitants (33 per cent of the population), show better results compared with HSC and VHSC MSUs of Fig. 8, discussed above. Although the 'Minimum-under-supply' is worst, the 'Mean-ofservice-status' and the 'Standard-deviation' are reduced dramatically, reflecting a better homogeneous response
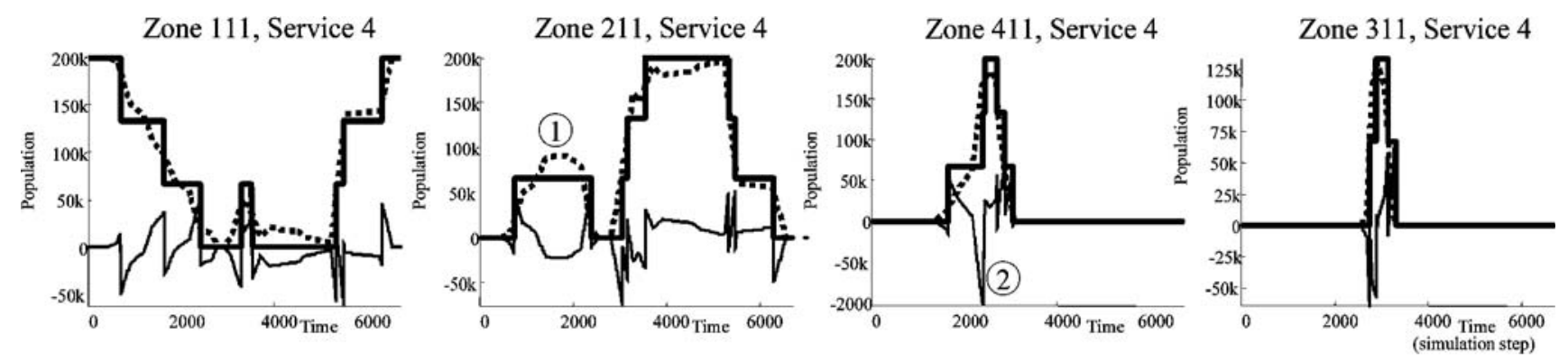

Zone 111, Service 5
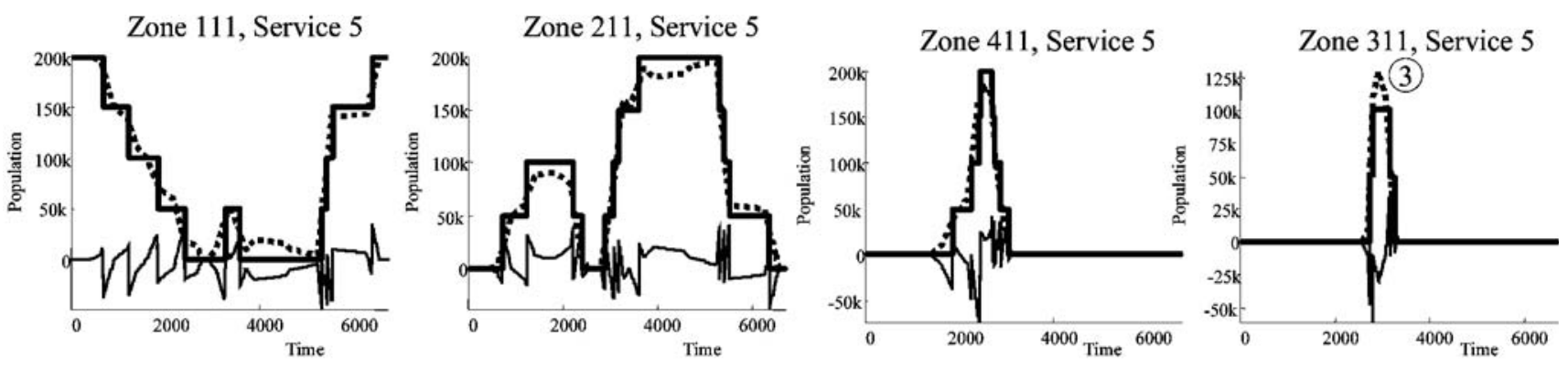

MSU Supply for service

- Service Status (MSU Supply - Demand)

Fig. 9 Response of the control for MSUs of medium service capacity 
for the control (Table 4). The 'Total-under-supply' is worse than those of HSC and VHSC MSUs but can still be acceptable relative to the service capacity for MSU group 4. The most important improvement is the need for no additional MSUs ( 0 per cent) compared with 50 and 300 per cent for HSC and VHSC MSUs respectively, which reflects higher efficiency and utilization of MSUs for the Hajj.

Another MSC MSU to be discussed is MSU group 5 shown in Fig. 9, row 2, which has a service capacity of 1000 PU (pilgrim unit) (25 per cent of population, i.e. 50000 inhabitants). By comparing it with MSU group 4 (Fig. 9, points 1 and 2), it can be seen that the response is improved as the supply curves match the demand curves better at zones 111, 211 and 411. This is due to the lower service capacity of MSU group 5, which gives the control four units to manipulate instead of three. Only at zone 311 does MSU group 5 show a region of under-supply (Fig. 9, point 3). This shortage is due to the low amount of extra demand but is not enough to justify sending additional MSUs of group 5, although it is already covered by the higher service capacity of MSU group 4.

It should be mentioned that any shortage of services at the beginning of demand curves may not be considered a serious problem since newly arrived pilgrims will spend some time settling down before they start looking for services. Also, a slight shortage of services, relative to the total capacity of existing MSUs, may not be considered a serious problem, since the existing MSUs can operate above their nominal capacity for short periods of time. This is usually acceptable in a service industry since service providers can handle the additional demand for services by operating above their nominal service capacity for short periods of time and customers usually accept that they will have to wait a bit longer to be served during peak times.

\subsection{MSUs of 'Low-service-capacity' and 'Very-low- service-capacity'}

Figure 10, row 1, represents the response of the control to the demand for MSU group 7. This group has a low service capacity (LSC) of $25 \mathrm{k}$ inhabitants ( 12.5 per cent of the maximum population); therefore, eight units/ batches are needed to fulfil the needs of the maximum population of any zone. From Fig. 10, it can be seen that the supply curve matches the demand curve better than HSC MSUs, especially for low demand peaks (Fig. 10, points 1 and 2). However, some delay in the response is noticed at the beginning of the demand curve in zone 311 (Fig. 10, point 3), which resulted in some shortage of service at that time. This delay is due to the high number of LSC MSUs needed to satisfy the increasing demand, while not all requests were approved due to the high traffic volume for transporting pilgrims from zone 411 to zone 311 during that period.

MSU group 9 has a very low service capacity (VLSC) of only $13.3 \mathrm{k}$ inhabitants ( 6.7 per cent of the maximum population). Initially, it was expected that such a low service capacity would provide the control with high flexibility to match any low variation in demand. However, due to the traffic limitations, not all requests for MSUs can be answered. Figure 10, row 2, shows the
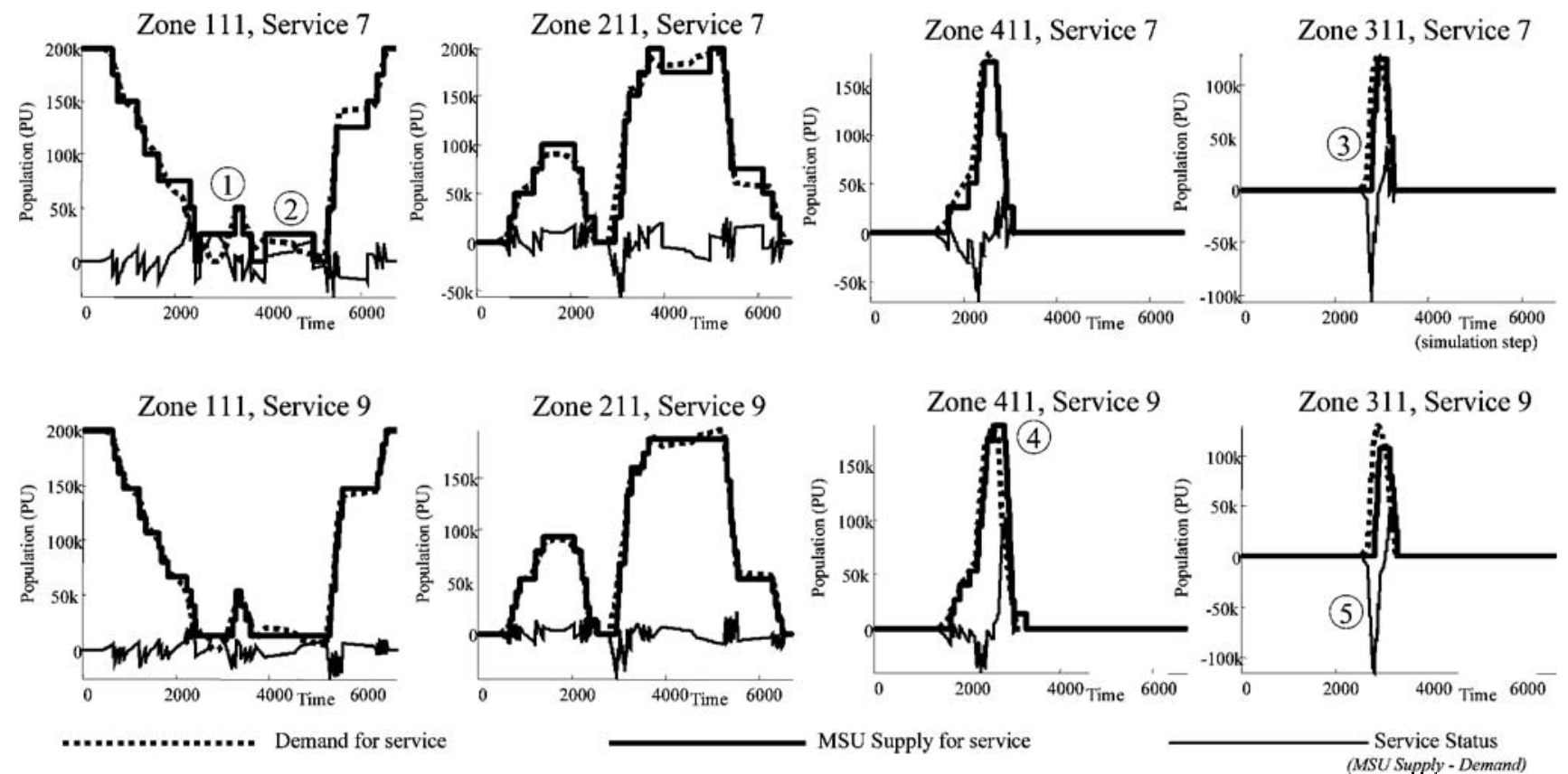

Fig. 10 Response of the control for MSUs of low and very-low service capacities 
good match between service demand and MSU supply in most zones, except at zone 311, where the delay for sending enough MSUs resulted in a severe shortage of service for a relatively long time (Fig. 10, point 5). Delays occurred also in releasing unneeded MSUs from zone 411 (Fig. 10, point 4), even though they were badly needed for zone 311, due to the high traffic volume at that period.

\section{CONCLUSION}

An approach for JIT-MSUs is shown as feasible to control the proposed system of MSUs for the Hajj, through an online control of the orders to move MSUs to provide the right services at the right place at the right time, achieving higher efficiency and utilization of resources.

Intelligent FLCs were used, on a microscopic scale, to evaluate the situations at every zone and to issue requests for MSUs. Another set of FLCs was used by the control centre to evaluate these requests from a macroscopic point of view in order to account for other conditions relating to the system as a whole. Since different zones compete for the same discrete limited resources, the control centre sorts these evaluated requests and issues orders according to their relative importance and the control parameters. This evaluation process, using relative scores, was found to be beneficial in managing/controlling MSUs and made the search for optimum settings for the FLCs subcritical since the same rules applied to all the zones/plants simultaneously. Modelling and simulation of the system of MSUs was found to be a very effective tool in developing the control rules and evaluating their effects on the level of services provided and traffic conditions without disturbing the mega-event of the Hajj.

MSUs of medium service capacity ranging from 50 to $66.7 \mathrm{k}$ inhabitants ( 25 to 33 per cent of the population) were found to be very suitable for use in the proposed system. They require no additional units, do not affect traffic volume significantly and were found to be stable to control. Lower service capacity MSUs down to $25 \mathrm{k}$ inhabitants are also suitable to some extent as they require no additional units and their performance can be improved by making use of external spaces in order to increase their service capacity, hence reducing the number of units. Very-high service capacity MSUs need to be reduced in their capacity and increased in their number of units to increase their efficiency. MSUs of very-low service capacity should be used sparingly, by increasing their service capacity, to allow for their transportation during peak times.

In general, the approach suggested by this paper, the analysis, the evaluation procedure and its recommendations can be applied to similar situations where different plants/systems are competing for the same resources/machines that can only be provided in high discrete values.

\section{ACKNOWLEDGEMENTS}

The authors are grateful for the financial support of the Custodian of the Two Holy Mosques Institute for Hajj Research, Umm Al-Qura University, Makkah, Saudi Arabia.

\section{REFERENCES}

1 Beeah, The Comprehensive Plan for Al-Mashaeer Almuqadasah, Elementary and Final Plan Report. The Central Committee for the Hajj, Makkah, Saudi Arabia, 1999.

2 Othman, F. M. A closed loop system for transporting pilgrims. In the Sixth Symposium for Transportation in the Hajj, Ministry of Transportation and King Abdul-AZIZ University, Saudi Arabia, 1991.

3 Othman, F. M. The system of ERSBS as a strategy for transporting pilgrims, goods, and services in Almasheer. In the Symposium for Transportation Planning in the Makkah Region, Makkah Region Committee and Municipality of Jeddah, Saudi Arabia, 1998.

4 Othman, F. and Parkin, R. M. A system of mobile service units (MSUs) for the large scale event industry. In the 8th Mechatronics Forum International Conference (Mechatronics 2002), University of Twente, The Netherlands, 24-26 June 2002.

5 King, R. E. Computational Intelligence in Control Engineering, 1999 (Marcel Dekker, New York).

6 Goser, K. and Surmann, H. Clever control systems designed quickly, users can construct fuzzy control systems for their own control problems. Elektronik, 17 March 1992, 60-61, 64-68.

7 Basu, S., Huynh, V. and Dutta, S. A formalism for modelling intelligent control of material handling equipment in a distribution centre. Int. J. Prod. Res. IEE, May 1996, 34(5), 1381-1398.

8 Driessen, B., Feddema, J. and Kwok, K. Decentralized fuzzy control of multiple nonholonomic vehicles. In Proceedings of the American Control Conference (ACC), Philadelphia, Pennsylvania, 24-26 June 1998, Vol. 1, pp. 404-410.

9 Wang, T. Development of a planning methodology for cellular manufacturing environments. $\mathrm{PhD}$, Cranfield, Bedford, 1996.

10 Feng, L. Control of large scale discrete event systems: task allocation and coordination. Systems and Control Lett., September 1991, 23(3), 169-175.

$11 \mathrm{Li}$, Y. and Gao, W. A new hierarchical coordination controller for large scale discrete event systems. In Proceedings of the 8th IEEE International Symposium on Intelligent Control, IEEE Control Systems Society, Chicago, Illinois, 25-27 August 1993, pp. 302-307. 
12 Othman, F. and Parkin, R. M. Modelling and simulation of a system of mobile service units (MSU) for the large scale event industry. In Proceedings of the International Conference on Mechatronics 2003, Loughborough University, Leicestershire, June 2003, pp. 97-102 (Professional Engineering Publishing, London and Bury St Edmunds).

13 Matia, F. and Al-Hadithi, B. Stability analysis for discrete fuzzy systems. In Proceedings of the 9th IEEE International Conference on Fuzzy Systems (FUZZ-IEEE 2000), San Antonio, Texas, 2000, Vol. 2, pp. 961-964.

14 Yamashita, T., Katoh, R., Singh, S. and Hori, T. Stability analysis of fuzzy control system applying conventional methods. In Proceedings of IEEE Instrumentation and
Control Engineering Conference, Kobe, Japan, 28 October1 November 1991, Vol. 2, pp. 1579-1584.

15 Johansen, T. Fuzzy model based control: stability, robustness, and performance issues. IEEE Trans. Fuzzy Systems, August 1994, 2, 221-234.

16 Malki, H., Huaidong, L. and Guanrong, C. New design and stability analysis of fuzzy proportional-derivative control systems. IEEE Trans. Fuzzy Systems, November 1994, 2(4), 245-254.

17 Cao, S., Rees, N. and Feng, G. Stability analysis of fuzzy control systems. IEEE Trans. Systems, Man, and Cybernetics, Part B: Cybernetics, February 1996, 201-204. 